\title{
Evidence-Based Clinical Practice Guidelines for the Management of Patients with Lentigo Maligna
}

\author{
Mitchell Robinson ${ }^{a, b} \quad$ Clare Primiero $^{a}$ Pascale Guitera ${ }^{c-f} \quad$ Angela Hong ${ }^{c, d, f}$ \\ Richard A. Scolyer ${ }^{c-f}$ Jonathan R. Stretch ${ }^{c, d, f}$ Geoffrey Strutton ${ }^{c, g}$ \\ John F. Thompson ${ }^{c-f}$ H. Peter Soyer ${ }^{a-c}$ \\ ${ }^{a}$ Dermatology Research Centre, The University of Queensland Diamantina Institute, The University of Queensland, \\ Brisbane, QLD, Australia; ${ }^{b}$ Department of Dermatology, Princess Alexandra Hospital, Brisbane, QLD, Australia; \\ ${ }^{\mathrm{C} C a n c e r}$ Council Australia Melanoma Guidelines Working Party, Sydney, NSW, Australia; ${ }^{\mathrm{d}}$ Melanoma Institute \\ Australia, The University of Sydney, Sydney, NSW, Australia; 'Department of Melanoma and Surgical Oncology, \\ Royal Prince Alfred Hospital, Sydney, NSW, Australia; 'Sydney Medical School, The University of Sydney, Sydney, \\ NSW, Australia; ${ }^{9}$ Department of Anatomical Pathology, Princess Alexandra Hospital, Brisbane, QLD, Australia
}

\section{Keywords}

Lentigo maligna · Melanoma · Guidelines · Surgical

margins - Radiotherapy · Reflectance confocal microscopy

\begin{abstract}
Introduction: Lentigo maligna (LM) is a subtype of melanoma in situ that usually occurs in sun-damaged skin and is characterised by an atypical proliferation of melanocytes within the basal epidermis. If left untreated, LM can develop into invasive melanoma, termed lentigo maligna melanoma, which shares the same prognosis as other types of invasive melanoma. The incidence rates of LM are steadily increasing worldwide, in parallel with increases in the incidence rates of invasive melanoma, and establishing appropriate guidelines for the management of $L M$ is therefore of great importance. Methods: A multidisciplinary working party established by Cancer Council Australia has recently produced up-to-date, evidence-based clinical practice guidelines for the management of melanoma and LM. Following selection of the most relevant clinical questions, a comprehensive literature
\end{abstract}

karger@karger.com

www.karger.com/drm

Karger $\stackrel{2}{\circ}$ search for relevant studies was conducted, followed by systematic review of these studies. Data were summarised and the evidence was assessed, leading to the development of recommendations. After public consultation and approval by the full guidelines working party, these recommendations were published on the Cancer Council Australia wiki platform (https://wiki.cancer.org.au/australia/Clinical_ question:Effective_interventions_to_improve_outcomes_ in_lentigo_maligna\%3F). Main Recommendations: Surgical removal of LM remains the standard treatment, with 5- to $10-\mathrm{mm}$ clinical margins when possible. While yet to be fully validated, the use of peri-operative reflectance confocal microscopy to assess margins should be considered where available. There is a lack of high-quality evidence to infer the most effective non-surgical treatment. When surgical removal of LM is not possible or refused, radiotherapy is recommended. When both surgery and radiotherapy are not appropriate or refused, topical imiquimod is the recommended treatment. Cryotherapy and laser therapy are not recommended for the treatment of LM.

(c) 2019 S. Karger AG, Basel

H. Peter Soyer

Dermatology Research Centre, The University of Queensland Diamantina Institute The University of Queensland

37 Kent Street, Woolloongabba QLD 4102 (Australia)

E-Mail p.soyer@uq.edu.au 


\section{Background}

Lentigo maligna (LM), historically known as Hutchinson's melanotic freckle, is a subtype of melanoma in situ usually occurring in sun-damaged skin and characterised by a proliferation of atypical melanocytes along the basal epidermis. If left untreated, LM can develop into invasive melanoma, termed lentigo maligna melanoma (LMM), which shares the same prognosis as other types of invasive melanoma. There are currently very limited data on the natural history of LM, with no longitudinal prospective studies performed to date. Based on an epidemiological study undertaken $>30$ years ago, Weinstock and Sober [1] reported that untreated LM in patients 65 years of age has a $1.2 \%$ chance of progressing to LMM in the following 10 years, with a lifetime risk of $2.2 \%$, while at 45 years of age patients have a $3.3 \%$ risk of progressing to LMM over the following 20 years, with a lifetime risk of $4.7 \%$. However, a recent review by Kasprzak and Xu [2] suggests that up to $30-50 \%$ of cases will progress to LMM if left untreated, with a latency period varying from 10 to 50 years. While the latency of LM to LMM is generally thought to be more than a decade, there have been reported cases of progression of LM to LMM in as little as 24 months [3]. LM usually occurs in the elderly and is most commonly found in the head or neck region on severely sun-damaged skin. However, LM is occasionally found on the trunk and extremities. The diagnosis of LM is based on clinical and dermoscopic features, confirmed by biopsy and histopathological assessment.

The most effective treatment of LM is complete surgical excision with at least 5-mm clinical margins, and preferably $10-\mathrm{mm}$ clinical margins. However, the anatomical location of the lesion, its size and the age of the patient can present challenges for surgical intervention. There are multiple non-surgical treatment alternatives currently in use including radiotherapy, cryotherapy, laser ablation and topical immunomodulatory therapies, such as imiquimod. These procedures have the advantage of reduced morbidity and cosmetic impact; however, they have not achieved the same level of complete lesion clearance and reduction in recurrence rates as surgical removal of lesions [4].

As the incidence rates of LM are steadily rising worldwide, with a recent Australian-based population study demonstrating that LM accounts for at least $45 \%$ of all diagnosed melanoma in situ, establishing appropriate clinical guidelines for its management is of great importance $[5,6]$. In Australia, the first national guidelines for the management of cutaneous melanoma and LM were published in 1999 by the Australian Cancer Network, with updated guidelines subsequently published in 2008 [7]. Due to the rapidly evolving nature of management options for melanoma, including LM, it was resolved in 2014 to update the national clinical practice guidelines once again, this time using an online wiki platform, through collaboration between the Cancer Council Australia and Melanoma Institute Australia, with support from the Skin Cancer College Australasia and the Australasian College of Dermatologists. The objective, as for the previous national guidelines, was to develop an up-todate, evidence-based set of recommendations and practice points to guide the best possible management of melanoma, including LM, in Australia. At present, the Cancer Council Australia melanoma wiki has 9 published chapters, one of which includes a section on LM. These guidelines address the key clinical aspects of melanoma management and act as the definitive, authoritative set of clinical guidelines in Australia.

\section{Methods}

A multidisciplinary working party established by Cancer Council Australia in partnership with Melanoma Institute Australia was assembled in November 2014 to produce and develop upto-date melanoma management guidelines based on current evidence within the literature (Table 1). Clinical questions were identified, including "What are the appropriate definitive clinical margins for excision of primary melanomas?" in which the subtype LM was included [8]. The methodological processes, levels of evidence and grades of recommendation employed for development of the guidelines are accessible at http://wiki.cancer.org.au/australia/Guidelines:Melanoma/Guideline_development_process. After public consultation, and review and approval by the members of the guidelines chapter, then ratification by the entire guidelines working party, these recommendations were published on the Cancer Council Australia wiki website (Table 2). Recommendations that did not meet strict evidence-based criteria but were relevant to practice were published as "practice points" to guide clinicians (Table 3 ).

\section{Systematic Review of Evidence Relating to LM}

Although surgical excision is the standard treatment recommendation for LM, there have to date been no randomised controlled trials (RCTs) that have compared the outcomes of surgical and non-surgical treatment methods. A recent cohort study of 33 patients with histologically confirmed LM described clearance in 21 cases managed over a mean period of 4.1 years with topical imiquimod [9]. While these findings were not statistically 
Table 1. Summary of evidence for lentigo maligna (LM)

\begin{tabular}{ll}
\hline Level \\
\hline
\end{tabular}

There have been no randomised controlled trials to n.a. date comparing the efficacy of all LM treatments

Mohs micrographic surgery has been shown to improve complete clearance rates and reduced recurrences over conventional surgical removal of LM [12-14]

Ultra-soft X-ray/grenzrays are a suitable alternative

III-1 to surgical excision of LM or as adjuvant therapy after surgical excision, especially for treatment of large lesions [15]

Radiotherapy has been shown to have superior IV complete clearance rates and few recurrences over imiquimod therapy for LM [15]

The addition of tazarotene to imiquimod as an IV adjuvant therapy can increase the inflammatory response when used to treat LM but it is unknown whether this translates into improved treatment efficacy [10]

The use of peri-operative reflectance confocal III-1, IV microscopy margin assessment of LM can aid in identifying subclinical extension and help define surgical excision margins [16-18]

There is currently a lack of sufficient evidence available to determine the efficacy of laser therapy $[4,21]$

The methodology, levels of evidence and grades of recommendation employed for guideline development are accessible at http://wiki.cancer.org.au/australia/Guidelines:Melanoma/Guideline_development_process.

significant, this study does highlight the role of topical imiquimod as an alternative to surgery for the treatment of LM in selected cases. One RCT that assessed the offlabel use of imiquimod $5 \%$ cream with or without tazarotene $0.1 \%$ gel for the treatment of LM was published by Hyde et al. [10]. This study concluded that the complete response rate of LM may be improved with the combined use of tazarotene and imiquimod; however, the trial did not achieve statistically significant results. In 2014, a Cochrane Systematic Review was conducted by Tzellos et al. [11] to compare all treatments of LM, though only the aforementioned imiquimod trial met the RCT inclusion criteria. The Cochrane review concluded that whilst the addition of tazarotene to imiquimod may increase the inflammatory response, it may also result in early cessation of therapy due to treatment-related side effects.

Evidence-Based Guidelines for the Management of Lentigo Maligna
Table 2. Evidence-based recommendations for lentigo maligna (LM)

\begin{tabular}{ll}
\hline & Grade \\
\hline $\begin{array}{ll}\text { Complete surgical removal of LM with 5- to 10-mm } \\
\text { clinical margins is the preferred management, when } \\
\text { possible }\end{array}$ & C \\
\hline
\end{tabular}

When available, peri-operative reflectance confocal C microscopy margin assessment should be considered

When surgical removal of LM is not possible or C refused, ultra-soft X-ray/grenzrays are recommended

When both surgery and radiotherapy of LM are not C appropriate or refused, imiquimod is recommended

Cryotherapy is not recommended for the treatment C of LM

Laser therapy is not recommended for the treatment C of LM

The methodology, levels of evidence and grades of recommendation employed for guideline development are accessible at http://wiki.cancer.org.au/australia/Guidelines:Melanoma/ Guideline_development_process.

Table 3. Practice points for lentigo maligna (LM)

Diagnosis of LM should be obtained by biopsy and histopathology Considering that the risk of LM evolving into invasive melanoma is low and generally takes many years, it may be more appropriate in selected cases with very elderly patients, or those with significant comorbidities, to monitor the lesion over time (watchful waiting) where follow-up is possible; if significant clinical or dermoscopic changes are detected, a biopsy in suspicious areas to confirm invasive disease should be performed

Three cohort studies comparing the outcomes of conventional surgical excision versus staged or Mohs micrographic surgery were identified for the guidelines update [12-14]. Conventional excision has historically aimed to achieve 5-mm clinical margins when lesion location permitted this. However, the studies reviewed suggested that 5 - $\mathrm{mm}$ clinical margins, originally recommended in the National Institutes of Health consensus statement in 1992, may be inadequate due to clinically indistinct peripheral tumour borders which are often associated with LM, contributing to recurrence rates of between 6 and $20 \%$ [12-14]. This highlights that histopathological assessment of surgical margins may be more informative 
than clinically measured margins. Mohs micrographic surgery has been advocated as a surgical method for LM removal. Mohs micrographic surgery has the advantage of intra-operative assessment of tumour margins, therefore conserving the amount of healthy tissue removed and has been reported to achieve lower recurrence rates $(0.5-6.3 \%)[14,15]$. The primary disadvantage of Mohs micrographic surgery, apart from its inconvenience and cost, remains the reliance on frozen sections and immunohistochemical staining for the challenging visualisation of epidermal melanocytes. Alternatively, staged excision, prompt formal histopathological assessment utilising paraffin-embedded sections, has been shown to improve the delineation of melanocytic neoplasia and associated atypia [13]. Delayed reconstruction after primary excision involves leaving an open wound requiring dressings while a carefully oriented, formalin-fixed paraffin-embedded sample is assessed and a formal pathology report issued. If close or positive margins are encountered, these can be re-excised. Definitive reconstruction is delayed until a satisfactory excision margin is achieved.

The use of reflectance confocal microscopy (RCM), a non-invasive imaging modality that allows for real-time in vivo quasi-histological analysis, to peri-operatively map the margins of LM in order to guide surgical excision has shown considerable promise. A prospective cohort study published by Yélamos et al. [16] in 2017 evaluating margins in 23 lesions with the use of handheld RCM radial videomosaic of the head and neck showed a good correlation with surgical defects after staged excision of LM/LMM. A similar study undertaken by Pellacani et al. [17] showed that peri-operative RCM margin assessment of LM/LMM accurately predicted negative histological margins in 21 of 23 lesions examined. In contrast, they noted that dermoscopically assisted clinical delineation was accurate in predicting precise tumour borders in only 6 of the observed cases. The role of RCM for the identification of subclinical LM spread has also been well established, with Guitera et al. [18] demonstrating in a series of 29 patients with dermoscopically visible LM that RCM identified subclinical disease evident $>5 \mathrm{~mm}$ beyond the dermoscopy margin in 17 cases. While larger studies are required to validate the use of RCM, the findings from these studies highlight the evolving role of peri-operative RCM margin assessment for LM.

In addition to the 2014 Cochrane Review, 3 other systematic reviews have assessed outcomes of non-surgical treatment of LM [4, 19, 20]. Mora et al. [19] and Tio et al. [20] assessed outcomes for patients treated with topical imiquimod by reviewing 45 and 41 studies, respectively. Both studies concluded that while surgical removal remains the gold standard for the treatment of LM, topical imiquimod is a potential option for those patients not eligible or not willing to undergo surgery and/or radiotherapy. Both reviews also recommended an intensive treatment regime of at least 60 applications, with a frequency of 6-7 applications per week. The clearance rates reported in both studies were $76-77 \%$ for histopathological clearance and $78 \%$ for clinical clearance, although both reviews were hindered by varying treatment protocols, short-term follow-up and risk of publication bias in the case reports reviewed.

In a systematic review by Read et al. [4] in 2016, 3 nonsurgical methods were evaluated: radiotherapy, topical imiquimod and laser therapy. The authors evaluated 29 studies and likewise concluded that surgical removal of LM remains the preferred treatment, but radiotherapy and topical imiquimod are both alternative treatment options, with radiotherapy achieving better complete response rates and fewer recurrences than imiquimod. Read et al. [4] also reported that the evidence supporting the use of laser therapy to treat LM was weak.

A cohort study published by Hedblad and Mallbris [15] in 2012 described the treatment of LM and early LMM with superficial energy radiotherapy in 593 patients. The authors assessed outcomes for 3 types of management: primary treatment with ultra-soft X-ray/grenzrays, partial surgical removal followed by ultra-soft X-ray/grenzrays and radical surgical excision followed by adjuvant postoperative ultra-soft X-ray/grenzrays, with reported complete clearance rates of 83,90 and $97 \%$, respectively. While radiotherapy has the advantage of being non-invasive, easy to perform, well tolerated and is associated with a positive cosmetic outcome, it achieves lower clearance rates and higher recurrence rates than surgical excision. Lee et al. [21] conducted a review of outcomes after treatment of LM by surgical excision, radiotherapy and carbon dioxide laser ablation. The authors found lower recurrence rates after surgical excision and carbon dioxide laser ablation; however, the difference was not statistically significant. A recent open-label efficacy trial conducted by Fikrle et al. [22] examined the use of 2,940-nm Er:YAG (erbium doped yttrium aluminium) laser therapy for the management of 17 histologically confirmed LM. The authors reported macroscopic clearance in all 17 cases following initial treatment, followed by 10 cases of confirmed histological clearance at 6-12 months. Three cases of histologically confirmed recurrence were reported over a 36-month period, with the remaining cases either lost to follow-up or refusing follow-up biopsy. While carbon dioxide laser ablation and 2,940-nm Er:YAG laser therapy 
may have a role in the treatment of LM when standard treatments are refused or unsuitable, there is currently only very weak evidence of their efficacy.

\section{Recommendations}

All publications reviewed by the multidisciplinary working party established by Cancer Council Australia indicated that the surgical removal of LM remains the standard treatment, with 5- to $10-\mathrm{mm}$ clinical margins when possible (Table 3). While yet to be fully validated, the use of peri-operative RCM margin assessment should be considered where available. There is a lack of highquality evidence to indicate the most effective non-surgical treatment. However, when surgical removal of LM is not possible or is refused, radiotherapy is recommended. When both surgery and radiotherapy of LM are not appropriate or refused, imiquimod is the recommended treatment. Cryotherapy and laser therapy are not recommended for the treatment of LM.

It is clear that further RCTs assessing treatment options other than surgery for LM patients are required. Currently a multisite, international RCT designated Radiotherapy or Imiquimod in Complex Lentigo Maligna (RADICAL) is under way, coordinated by the Australia and New Zealand Melanoma Trials Group, to compare outcomes of radiotherapy versus imiquimod for complex LM where surgery is considered inappropriate or refused. This trial is expected to produce a strong level of evidence that may influence future guidelines for the non-surgical treatment of LM. Meanwhile surgical excision with 5- to 10 -mm clinical margins remains the standard, evidencebased treatment recommendation.

\section{Key Message}

Complete surgical removal with 5- to 10 -mm clinical margins is the preferred management of lentigo maligna; however, when surgical removal is not possible or refused, radiotherapy is recommended.

\section{Acknowledgments}

The authors would like to acknowledge the Cancer Council Australia, the Melanoma Institute of Australia, the Australasian College of Dermatology and the Skin Cancer College Australasia for their contributions in the development of these up-to-date practice guidelines for the management of patients with lentigo maligna.

\section{Statement of Ethics}

The authors have no ethical conflicts to disclose.

\section{Disclosure Statement}

The authors have no conflicts of interest to declare.

\section{Funding Sources}

Cancer Council Australia and Melanoma Institute Australia contributed in kind resources consisting of project staff, facilities, systems and travel budget to revise previous guidelines. The Skin Cancer College Australasia provided funding to enable employment of an additional full-time Project Officer in the Systematic Review team.

\section{Author Contributions}

M.R. and C.P.: substantial contributions to the literature review, evidence interpretation, provisional and primary drafting, in addition to revising paper critically for all important intellectual content. P.G., A.H., R.A.S., J.R.S. and G.S.: substantial contributions to the literature review, evidence interpretation, revising the paper critically for all important intellectual content (member of the Cancer Council Australia lentigo maligna management guideline development subcommittee). J.F.T.: substantial contributions to the literature review, evidence interpretation, provisional and primary drafting and revising the paper critically for all important intellectual content (member of the Cancer Council Australia lentigo maligna management guideline development subcommittee). H.P.S.: substantial contributions to the literature review, evidence interpretation, provisional and primary drafting, in addition to revising the paper critically for all important intellectual content (member of the Cancer Council Australia lentigo maligna management guideline development subcommittee).

\section{References}

Evidence-Based Guidelines for the

Management of Lentigo Maligna
1 Weinstock MA, Sober AJ. The risk of progression of lentigo maligna to lentigo maligna melanoma. Br J Dermatol. 1987 Mar;116(3):303-10.

2 Kasprzak JM, Xu YG. Diagnosis and management of lentigo maligna: a review. Drugs Context. 2015 May;4:212281.

3 Michalik EE, Fitzpatrick TB, Sober AJ. Rapid progression of lentigo maligna to deeply inva- sive lentigo maligna melanoma. Report of two cases. Arch Dermatol. 1983 Oct;119(10):8315.

4 Read T, Noonan C, David M, Wagels M, Foote M, Schaider H, et al. A systematic review of non-surgical treatments for lentigo maligna. J Eur Acad Dermatol Venereol. 2016 May;30(5):748-53. 
5 Guitera P, Collgros H, Madronio CM, Goumas C, Mann GJ, Watts CG, et al. The steadily growing problem of lentigo maligna and lentigo maligna melanoma in Australia: population-based data on diagnosis and management. Australas J Dermatol. 2019 May;60(2): $118-25$.

6 Matas-Nadal C, Malvehy J, Ferreres JR, Boada A, Bodet D, Segura S, et al. Increasing incidence of lentigo maligna and lentigo maligna melanoma in Catalonia. Int J Dermatol. 2019 May;58(5):577-81.

7 Cancer Council Australia Melanoma Guidelines Working Party. Clinical practice guidelines for the diagnosis and management of melanoma. Sydney: Cancer Council Australia. Available from: https://wiki.cancer.org. au/australiawiki/index.php?oldid=201397 . 2019.

8 Soyer HP, Guitera P, Hong A, Scolyer R, Stretch J, Strutton G, Party CCAMGW: What are the moset effective treatment/managment interventions to improve outcomes in patients with lentigo maligna, 2018.

9 Papanikolaou M, Lawrence CM: Long-term outcomes of imiquimod-treated lentigo maligna. Clin Exp Dermatol. 2019 Aug;44(6): 631-6.

10 Hyde MA, Hadley ML, Tristani-Firouzi P, Goldgar D, Bowen GM. A randomized trial of the off-label use of imiquimod, $5 \%$, cream with vs without tazarotene, $0.1 \%$, gel for the treatment of lentigo maligna, followed by conservative staged excisions. Arch Dermatol. 2012 May;148(5):592-6.
11 Tzellos T, Kyrgidis A, Mocellin S, Chan AW, Pilati P, Apalla Z. Interventions for melanoma in situ, including lentigo maligna. Cochrane Database Syst Rev. 2014 Dec;(12):CD010308.

12 Walling HW, Scupham RK, Bean AK, Ceilley RI. Staged excision versus Mohs micrographic surgery for lentigo maligna and lentigo maligna melanoma. J Am Acad Dermatol. 2007 Oct;57(4):659-64.

13 Hilari H, Llorca D, Traves V, Villanueva A, Serra-Guillen C, Requena C, et al. Conventional surgery compared with slow Mohs micrographic surgery in the treatment of lentigo maligna: a retrospective study of 62 cases. Actas Dermosifiliogr. 2012 Sep; 103(7):614-23.

14 Hou JL, Reed KB, Knudson RM, Mirzoyev SA, Lohse CM, Frohm ML, et al. Five-year outcomes of wide excision and Mohs micrographic surgery for primary lentigo maligna in an academic practice cohort. Dermatol Surg. 2015 Feb;41(2):211-8.

15 Hedblad MA, Mallbris L. Grenz ray treatment of lentigo maligna and early lentigo maligna melanoma. J Am Acad Dermatol. 2012 Jul; 67(1):60-8.

16 Yélamos O, Cordova M, Blank N, Kose K, Dusza SW, Lee E, et al. Correlation of Handheld Reflectance Confocal Microscopy With Radial Video Mosaicing for Margin Mapping of Lentigo Maligna and Lentigo Maligna Melanoma. JAMA Dermatol. 2017 Dec;153(12):1278-84.

17 Pellacani G, De Carvalho N, Ciardo S, Ferrari B, Cesinaro AM, Farnetani F, et al. The smart approach: feasibility of lentigo maligna superficial margin assessment with hand-held reflectance confocal microscopy technology. J Eur Acad Dermatol Venereol. 2018 Oct; 32(10): 1687-94.

18 Guitera P, Moloney FJ, Menzies SW, Stretch JR, Quinn MJ, Hong A, et al. Improving management and patient care in lentigo maligna by mapping with in vivo confocal microscopy. JAMA Dermatol. 2013 Jun;149(6): 692-8.

19 Mora AN, Karia PS, Nguyen BM. A quantitative systematic review of the efficacy of imiquimod monotherapy for lentigo maligna and an analysis of factors that affect tumor clearance. J Am Acad Dermatol. 2015 Aug; 73(2):205-12.

20 Tio D, van der Woude J, Prinsen CA, Jansma EP, Hoekzema R, van Montfrans C. A systematic review on the role of imiquimod in lentigo maligna and lentigo maligna melanoma: need for standardization of treatment schedule and outcome measures. J Eur Acad Dermatol Venereol. 2017 Apr;31(4):61624.

21 Lee H, Sowerby LJ, Temple CL, Yu E, Moore CC. Carbon dioxide laser treatment for lentigo maligna: a retrospective review comparing 3 different treatment modalities. Arch Facial Plast Surg. 2011 Nov-Dec;13(6): 398-403.

22 Fikrle T, Divišová B, Šuchmannová J, Pizinger K. The use of 2940-nm ER:YAG laser for the treatment of lentigo maligna. J Dtsch Dermatol Ges. 2019 Apr;17(4):425-31. 